\title{
Avaliação de compósitos híbridos para aplicações em Engenharia
}

\section{Hybrid composites evaluation to be applied in Engineering}

Juliana Marques Resende ${ }^{1}$

Artigo

Franciny Lima de Oliveira ${ }^{1}$

Original

Daniella Regina Mulinari²

Original

Paper

Palavras-chave:

Compósitos híbridos

Fibra proveniente da coroa do abacaxi

Polipropileno

\section{Resumo}

Os compósitos termoplásticos reforçados com fibras de vidro têm sido utilizados na indústria automobilística. No entanto, esses compósitos são relativamente caros e abrasivos aos equipamentos. Neste trabalho, foram avaliadas as propriedades mecânicas dos compósitos híbridos de polipropileno reforçados com fibras de vidro e fibras provenientes da coroa do abacaxi tratadas com solução alcalina. A morfologia das fibras foi analisada por microscopia eletrônica de varredura (MEV). Os resultados obtidos mostraram que os compósitos híbridos apresentaram propriedades mecânicas intermediárias comparadas aos demais compósitos.

\begin{abstract}
Thermoplastic composites reinforced with glass fibers have been used in the automobile industry. However, these composites are relatively expensive and abrasive to the equipment. In this work, the mechanical properties of the hybrid composites of polypropylene reinforced with glass fibers and pineapple crown fibers treated with alkaline solution were evaluated. The morphology of the treated fibers was analyzed by scanning electronic microscopy (SEM). The results showed that the hybrid composites presented intermediate mechanical properties compared to the other composites.
\end{abstract}

Key words:

Hybrid composites

Pineapple crown fibers

Polypropylene
Recebido em 03/2011

Aprovado em $04 / 2011$ 


\section{Introdução}

Atualmente as indústria têm substituído os materiais tradicionais por materiais poliméricos. Os polímeros têm demonstrado um alto grau de confiabilidade e muitas vantagens sobre os materiais convencionais; além de maior flexibilidade de projeto e economia na produção, pois sua baixa densidade é essencial para a redução do consumo de combustíveis ${ }^{1}$.

Aproximadamente, para $100 \mathrm{~kg}$ de polímeros empregados em um veículo, 200 a 300 $\mathrm{kg}$ de outros materiais deixam de ser empregados, refletindo no peso final do carro.

Dessa forma, estimando-se a vida útil de um veículo em 150.000 quilômetros, pode-se economizar 750 litros de combustível devido ao uso de plásticos. Além disso, a utilização de polímeros favorece a injeção de peças complexas com alto nível de produção e qualidade sem falar na resistência à corrosão ${ }^{1}$.

Nesse contexto, pesquisadores têm buscado o desenvolvimento de novos materiais, capazes de conciliar o alto desempenho dos polímeros de engenharia com a questão ecológica.

Dessa forma, o uso de materiais compósitos tem aumentado a cada dia, devido às melhorias das características dos materiais, isto é, das propriedades da matriz polimérica, do reforço fibroso e da interface polímero-reforço ${ }^{2}$.

As fibras naturais têm sido bastante investigadas para uso como reforço em compósitos de matrizes poliméricas, pois aliam propriedades que levam em consideração aspectos que vão de encontro a nova ordem mundial, de forte apelo ecológico. Além disso, as fibras naturais apresentam outras características interessantes tais como, baixo custo, baixa densidade, são provenientes de fontes renováveis, são biodegradáveis, não são tóxicas e possuem alto módulo específico, o que as tornam fortes candidatas em potencial para estas aplicações de engenharia ${ }^{3-8}$.

Dentre as diversas fibras naturais, as fibras provenientes da coroa do abacaxi vêm como uma alternativa viável e abundante quando comparado às fibras sintéticas que apresentam custo elevado e são provenientes de fontes não renováveis ${ }^{9-11}$.

O Brasil é um grande produtor agrícola e, portanto, essas fibras são geradas em grande quantidade através do descarte residual dos excedentes da fruta. O abacaxi, por exemplo, está entre as frutas mais produzidas e consumidas no Brasil, na qual a coroa é um excedente que não apresenta nenhuma utilidade econômica.

Luo e colaboradores (2004) avaliaram as propriedades mecânicas dos compósitos obtidos a partir da resina poli (3-hidroxibutiratocovalerato) reforçada com 20 e $30 \%$ de fibras de abacaxi e obtiveram resultados de resistência a tração e flexão altamente elevada quando comparados à resina pura ${ }^{12}$.

Arib e colaboradores (2006) também avaliaram as propriedades mecânicas dos compósitos de polipropileno reforçados com fibras de abacaxi e obtiveram um aumento significativo da resistência à tração e à flexão quando comparado à matriz de polipropileno pura ${ }^{13}$.

Nesse contexto, a proposta deste trabalho foi avaliar utilização dessas fibras na fabricação de um compósito híbrido. Os compósitos foram produzidos por meio da mistura física das fases dispersas na forma de fibras (coroa do abacaxi e vidro) na fase contínua, o polipropileno. Também foram produzidos compósitos de polipropileno reforçados com fibras de vidro e reforçados com fibras da coroa do abacaxi.

\section{Materiais e Métodos}

A metodologia desenvolvida para o cumprimento dos objetivos propostos neste trabalho foi otimizada e realizada para avaliar a viabilidade técnico-científica da proposta.

Para a confecção do compósito foram utilizadas fibras provenientes da coroa do abacaxi, fibras de vidro e polipropileno.

As fibras foram obtidas a partir da coroa descartada na comercialização da fruta do abacaxi. Após a retirada das fibras da coroa do abacaxi, a mesma foi seca em estufa a $50^{\circ} \mathrm{C}$ por 48 horas, a fim de remover a umidade. Em seguida as fibras sofreram processos físicos de trituração e peneiração, utilizando um liquidificador convencional e uma peneira de 25 mesh.

O procedimento realizado para o tratamento da fibra foi a imersão das fibras em uma solução de $\mathrm{NaOH}$ à $10 \%$ durante 1 hora e em temperatura ambiente. Após esse tempo, as fibras foram lavadas exaustivamente com água 
destilada até atingir o $\mathrm{pH}$ da água destilada e secas em estufa a $80^{\circ} \mathrm{C}$.

O polipropileno (PP) utilizado neste trabalho foi fornecido pela BRASKEN. O PP a ser utilizado é indicado para peças injetadas que possui excelente resistência mecânica e ao calor e alta resistência ao impacto.

\subsection{Análise da Modificação das Fibras}

As fibras provenientes da coroa do abacaxi in natura e modificadas foram caracterizadas por Microscopia eletrônica de varredura (MEV). As micrografias foram obtidas em um microscópio eletrônico de varredura JEOL JSM5310, disponível no INPE em São José dos Campos, com filamento de tungstênio operando a $10 \mathrm{kV}$, usando elétrons secundários, a fim de obter informações quanto à morfologia e composição das fibras.

\subsection{Obtenção dos Compósitos}

Os compósitos foram obtidos em um homogeneizador de plásticos de laboratório da MH Equipamentos (modelo MH-50H), disponível na Divisão de Materiais (AMR) do Comando-Geral de Tecnologia Aeroespacial (CTA).

A mistura entre as fibras (vidro e abacaxi modificadas) e o polímero ocorreu dentro da cápsula bi-partida, com arrefecimento de água. As palhetas de homogeneização rotacionaram com aproximadamente $2600 \mathrm{rpm}$ na primeira velocidade e $5250 \mathrm{rpm}$ na segunda, tornando o processo de homogeneização extremamente rápido. A primeira velocidade serviu para tirar o motor e o eixo do ponto de inércia e a segunda para misturar os materiais. E foi nesse momento que o equipamento foi desligado para que não ocorresse a degradação do material.

Para a obtenção dos compósitos, primeiramente as fibras foram secas em estufa a $50^{\circ} \mathrm{C}$ por 3 h. O PP também foi seco á mesma temperatura, porém, por $1 \mathrm{~h}$. Posteriormente, os materiais foram pesados nas proporções de $2,5 \%$ $(\mathrm{m} / \mathrm{m})$ de fibras de vidro e fibras provenientes da coroa do abacaxi modificadas. Foram preparados cerca de $250 \mathrm{~g}$ do material compósito em bateladas de $50 \mathrm{~g}$ no homogeneizador de plásticos. Após a mistura, o material fundido passou entre rolos de aços inox (calandras), seguido de resfriamento com imersão em água. Após a imersão em água, os compósitos foram moídos em moinho granulador (RONE) até passar por peneira de $13 \mathrm{~mm}$ e secos em estufa a $50^{\circ} \mathrm{C}$ por $3 \mathrm{~h}$.

Os compósitos moídos, previamente secos, foram injetados em molde contendo cavidades com dimensões específicas para ensaios mecânicos, utilizando uma Injetora Battenfeld HM 60/350, disponível no Centro Universitário da FEI.

Também foram preparados compósitos de polipropileno, reforçados com 5\% de fibras de vidro e compósitos de polipropileno, reforçados com 5\% de fibras provenientes da coroa do abacaxi para efeito de comparação. Os compósitos obtidos e tipos de fibras estão relacionados na Tabela 1.

Tabela 1. Descrição dos compósitos

\begin{tabular}{|c|c|c|c|c|}
\hline Amostra & $\begin{array}{l}\text { Tipo de fibra } \\
\text { reforçada }\end{array}$ & $\begin{array}{c}\text { Quantidade } \\
\text { de PP } \\
(\% \mathrm{~m} / \mathrm{m})\end{array}$ & $\begin{array}{c}\text { Quantidade } \\
\text { de reforço } \\
\text { (abacaxi) } \\
(\% \mathrm{~m} / \mathrm{m})\end{array}$ & $\begin{array}{l}\text { Quantidade de } \\
\text { reforço (vidro) } \\
\text { (\% m/m) }\end{array}$ \\
\hline $\mathrm{CH}$ & $\begin{array}{l}\text { Fibras de vidro / } \\
\text { Fibra do abacaxi }\end{array}$ & 95 & 2,5 & 2,5 \\
\hline $\mathrm{CV}$ & Fibras de vidro & 95 & -- & 5 \\
\hline $\mathrm{CA}$ & Fibras do abacaxi & 95 & 5 & -- \\
\hline
\end{tabular}




\subsection{Propriedades Mecânicas}

As propriedades mecânicas foram avaliadas por meio de ensaios mecânicos de tração e impacto.

Os ensaios de tração foram realizados no Laboratório de Ensaios Mecânicos do Centro Universitário de Volta Redonda - UniFOA, em um equipamento da marca EMIC (Figura 1).

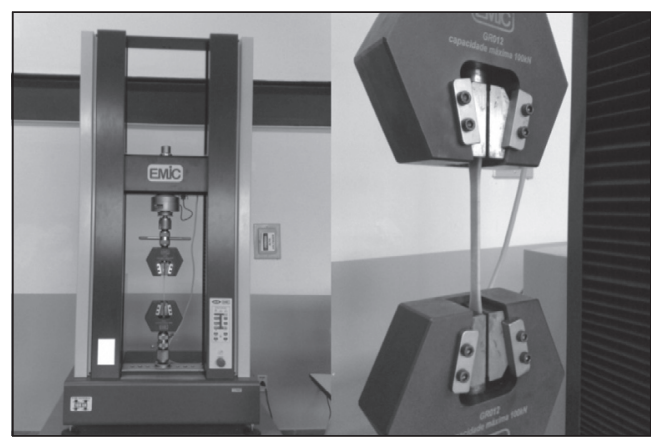

Figura 1. Máquina EMIC utilizada para o ensaio de tração.

Para cada tipo de compósito avaliado, foram ensaiados cinco corpos de prova com dimensões de acordo com a norma ASTM D 638 - 03 com $13 \mathrm{~mm}$ de largura, $165 \mathrm{~mm}$ de comprimento e $3 \mathrm{~mm}$ de espessura. As propriedades mecânicas de resistência à tração, alongamento e módulo de elasticidade em tração foram avaliadas.

\section{Resultados e Discussão}

\subsection{Microscopia Eletrônica de Varredura (MEV)}

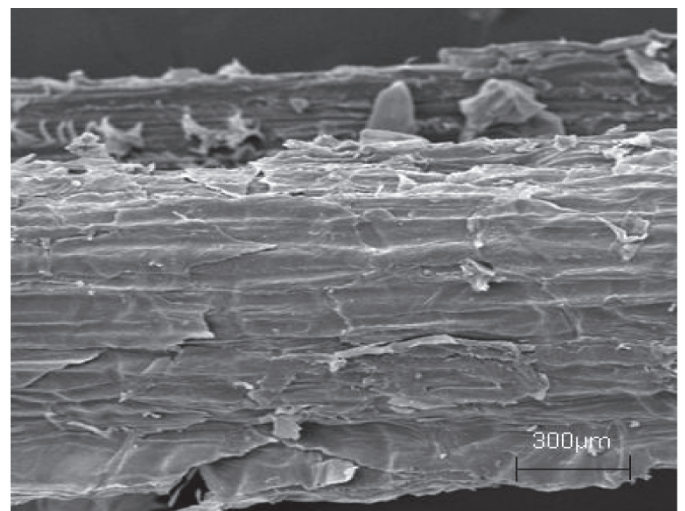

Os ensaios de impacto foram realizados no Laboratório de Ensaios Mecânicos do Centro Universitário de Volta Redonda UniFOA, utilizando o equipamento da marca PANTEC (Figura 2).

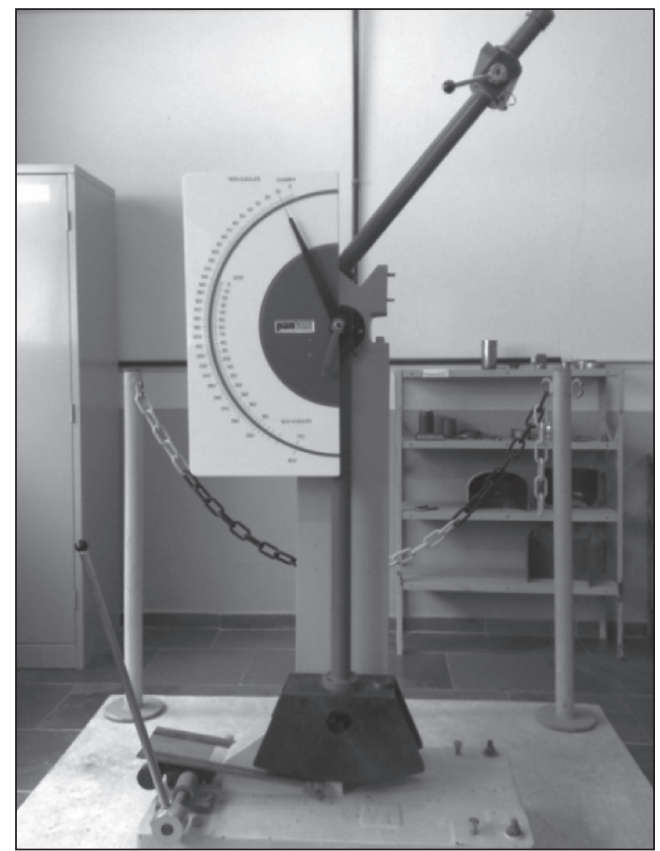

Figura 2. Máquina PANTEC utilizada para o ensaio de impacto.

Foram analisados cinco corpos de prova, com dimensões de acordo com a norma ASTM D 6110 06 com $12 \mathrm{~mm}$ de largura, $63,5 \mathrm{~mm}$ de comprimento e $12 \mathrm{~mm}$ de espessura. Foram avaliadas a energia absorvida ao impacto e a resistência.

A Figura 3 evidencia as micrografias obtidas das fibras provenientes da coroa de abacaxi in natura e modificadas.

Figura 3. Micrografias das fibras de abacaxi: (A) fibra in natura 500X; (B) fibra modificada 500X.

A micrografia da fibra de abacaxi in natura (Figura 3A) revelou fragmentos achatados dispostos de forma ordenada. Observouse uma superfície lisa e homogênea, devido à presença dos extrativos, assemelhando-se a

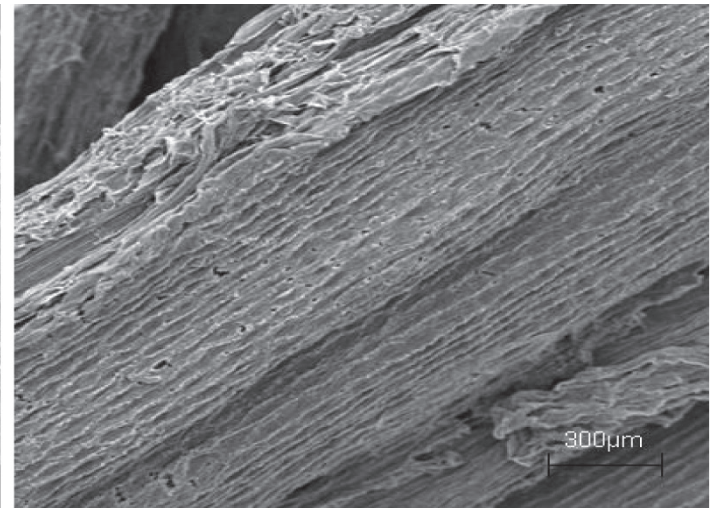

uma cera, que ainda estão presentes nas fibras. A análise da fibra após a modificação (Figura 3B) revelou o aparecimento da superfície rugosa, devido à modificação com $\mathrm{NaOH}$, que promoveu uma desagregação das fibras em 
microfibrilas, que visualmente torna as fibrilas mais expostas. Observou-se também a presença de poros ou orifícios presentes em toda a superfície rugosa da fibra indicando que poderá ocorrer um aumento da área superficial efetiva para o contato com a matriz polimérica.

\subsection{Propriedades Mecânicas}

riais está fundamentado na viscoelasticidade. O termo viscoelasticidade está associado à resposta elástica e viscosa, simultânea ou não, apresentada pelos polímeros. Um material viscoelástico, sob deformação, terá um comportamento misto que resultará em recuperação parcial da deformação da deformação depois de retirada a força que atua sobre este ${ }^{14}$.

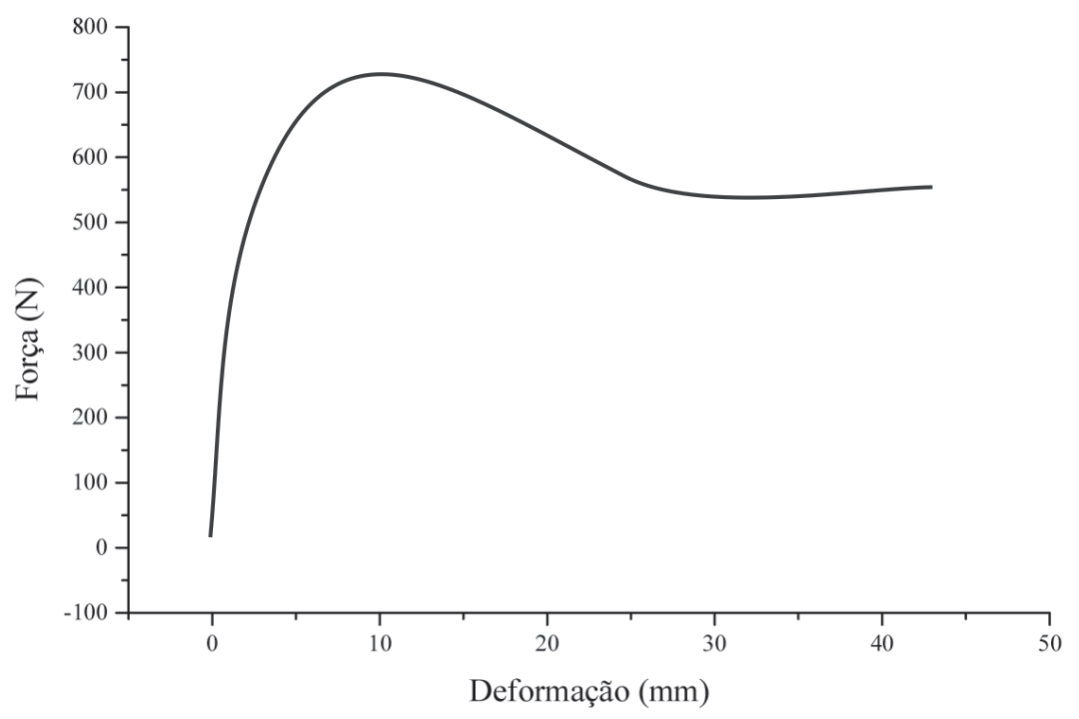

Figura 4. Comportamento dos materiais no ensaio de tração.

A Tabela 2 apresenta os valores do limite de resistência à tração e seus respectivos alongamento e módulo, obtidos dos ensaios mecânicos dos compósitos PP reforçados com as fibras de vidro, PP reforçados com as fibras provenientes da coroa do abacaxi tratadas com $\mathrm{NaOH} 1 \% \mathrm{~m} / \mathrm{v}$, PP reforçados com fibras de vidro e fibras prove- nientes da coroa do abacaxi tratadas com $\mathrm{NaOH}$ $1 \% \mathrm{~m} / \mathrm{v}$ e também do polipropileno puro.

Analisando-se os resultados da Tabela 2 observou-se um aumento no módulo de elasticidade ao inserir reforço na matriz polimérica. Por outro lado, a resistência praticamente manteve-se a mesma.

Tabela 2. Valores do limite de resistência à tração.

\begin{tabular}{|c|c|c|c|}
\hline \multirow[b]{2}{*}{ Amostras } & \multicolumn{3}{|c|}{ Propriedades } \\
\hline & $\begin{array}{c}\text { Alongamento no } \\
\text { limite de resistência } \\
\text { à tração }(\%)\end{array}$ & $\begin{array}{c}\text { Limite de resistência } \\
\text { à tração (MPa) }\end{array}$ & $\begin{array}{c}\text { Módulo de } \\
\text { Elasticidade (MPa) }\end{array}$ \\
\hline PP & $1,43 \pm 0,2$ & $22,2 \pm 0,04$ & $22,2 \pm 0,04$ \\
\hline $\mathrm{CV}$ & $1,00 \pm 0,03$ & $22,02 \pm 0,06$ & $22,02 \pm 0,06$ \\
\hline $\mathrm{CA}$ & $0,87 \pm 0,03$ & $0,87 \pm 0,03$ & $2508,9 \pm 58,8$ \\
\hline $\mathrm{CH}$ & $0,80 \pm 0,03$ & $0,80 \pm 0,03$ & $2671,2 \pm 66,4$ \\
\hline
\end{tabular}


No entanto, o compósito híbrido apresentou maior rigidez quando comparado aos demais. Este fato ocorreu, devido ao sinergismo das fibras de vidro e fibras provenientes da coroa do abacaxi tratadas.

\subsubsection{Resistência ao Impacto}

Dentre as propriedades mecânicas dos compósitos avaliadas neste trabalho, à resistência ao impacto mostrou maior relevância, pois a avaliação da resistência ao impacto dos materiais é um fator importante na seleção de materiais para aplicações de engenharia (MULINARI, 2009).

Analisando-se a resistência ao impacto das amostras, foram observados que os compósitos apresentaram maiores valores, quando comparados ao PP puro, atingindo até $108,05 \%$ de aumento (CV). Esse aumento na resistência ocorreu devido à inserção de fibras na matriz, fazendo com que a energia absorvida aumentasse e, consequentemente, a resistência. Os resultados obtidos de resistência ao impacto podem ser observados na Tabela 3.

Tabela 3. Valores do limite de resistência ao impacto

\begin{tabular}{|c|c|c|}
\hline Amostras & Energia Absorvida (J) & Resistência (J.m-1) \\
\hline PP puro & $6,0 \pm 0,6$ & $36,0 \pm 1,1$ \\
\hline CV & $11,33 \pm 2,3$ & $74,9 \pm 15,3$ \\
\hline CA & $6,75 \pm 0,96$ & $43,05 \pm 6,2$ \\
\hline CH & $8,0 \pm 2,0$ & $51,3 \pm 12,7$ \\
\hline
\end{tabular}

Os compósitos reforçados com fibras de vidro apresentaram maiores resistências, quando comparados aos compósitos reforçados com fibras provenientes da coroa do abacaxi e aos compósitos híbridos. No entanto, os compositos híbridos, ou seja, aqueles reforçados com fibras de vidro e abacaxi apresentaram energia absorvida e resistência ao impacto intermediária aos demais compósitos. As Figuras 5 e 6 evidenciam estes resultados.

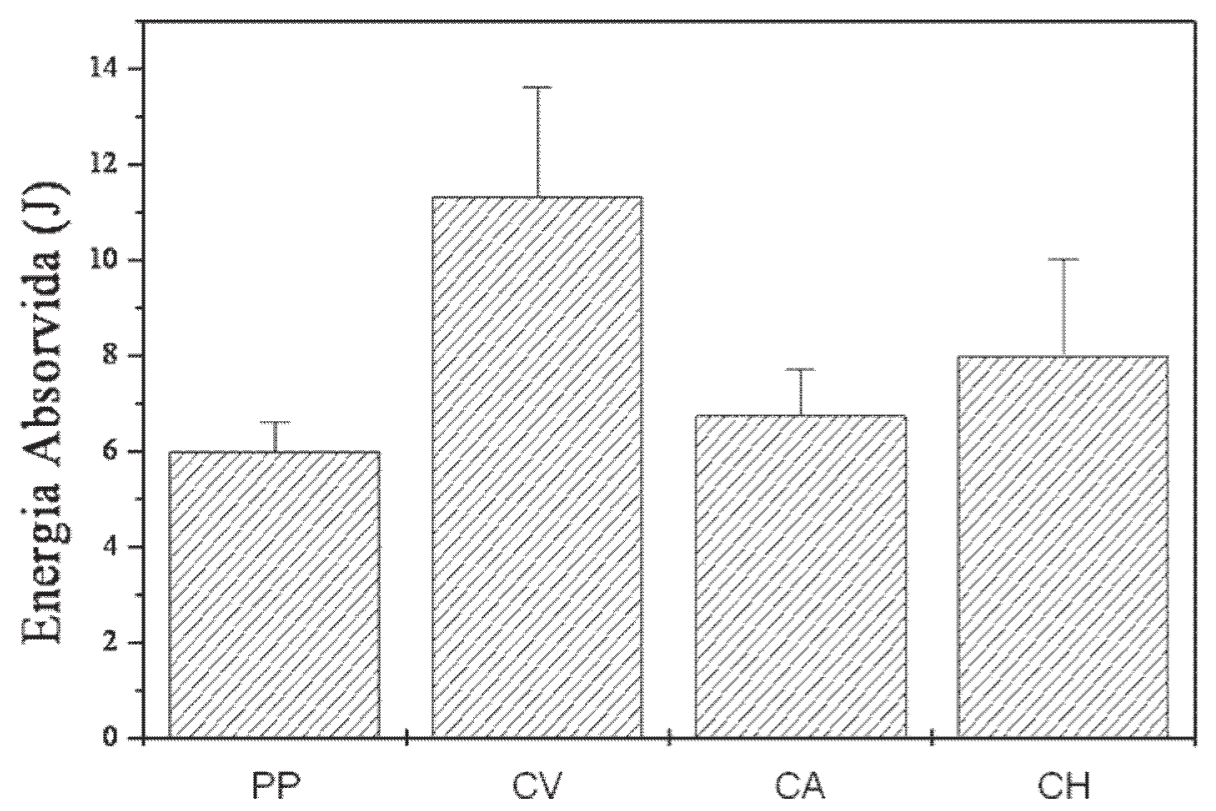

Figura 5. Energia absorvida do polipropileno puro e dos compósitos. 


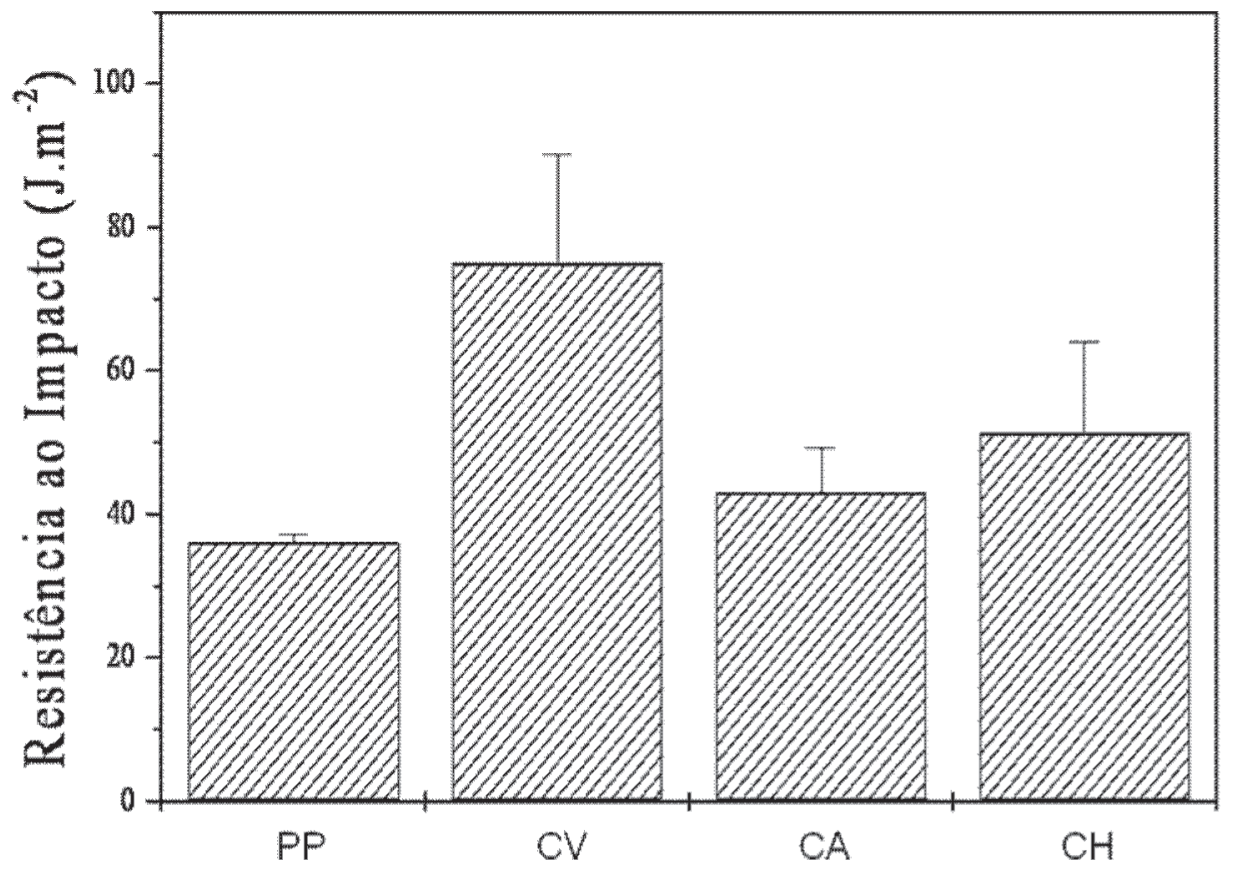

Figura 6. Resistência ao impacto do polipropileno e dos compósitos.

Santos (2006) apresentou resultados similares ao estudar compósitos híbridos reforçados com fibras de vidro e fibras de coco tratadas com solução alcalina.

A inserção de fibras na matriz também aumentou a resistência ao impacto, devido ao mecanismo de dissipação de energia. As fibras foram arrancadas (pull out) da matriz e dissiparam energia durante o processo de fricção mecânica. E esse arrancamento das fibras preveniu a concentração de tensão nas áreas ao longo da fibra.

\section{Conclusões}

As fibras provenientes da coroa do abacaxi apresentaram alterações significativas em sua morfologia quando submetidas ao tratamento alcalino. Com a inserção dessas fibras tratadas na matriz de polipropileno também reforçada com fibra de vidro, notou-se que os compósitos híbridos apresentaram propriedades mecânicas intermediárias quando comparada aos compósitos reforçados com fibras de vidro e aos compósitos reforçados com fibras provenientes da coroa do abacaxi tratadas.

\section{Referências Bibliográficas}

1. SANTOS, A. M. Estudo de compósitos híbridos polipropileno / fibras de vidro e coco para aplicações em engenharia. Dissertação (Mestre em Engenharia Mecânica) - Universidade Federal do Paraná, Curitiba, 2004.

2. WAMBUA, P.; IVENS, J.; VERPOEST, I. Natural fibres: Can they replace glass in fibre reinforced plastics? Composites Science and Technology, v. 63, p. 12591264, 2003.

3. CARVALHO, K.C.C.; MULINARI, D.R.; VOORWALD, H.J.C.; CIOFFI, M.O.H. Chemical modification effect on the mechanical properties of HIPS/ coconut fiber composites. BioResources, v.5 (2), p.1143-1155, 2010.

4. NIRMAL, U.; YOUSIF, B. F.; RILLING, D.; BREVERN, P. V. Effect of betelnut fibres treatment and contact conditions on adhesive wear and frictional performance of polyester composites. Wear, v.268, p.1354-1370, 2010. 
5. MULINARI, D. R.; VOORWALD, H. J. C.; CIOFFI, M. O. H.; ROCHA, G. J. M., DA SILVA, M. L. P. Surface modification of sugarcane bagasse cellulose and its effect on mechanical and water absorption properties of sugarcane bagasse cellulose/ HDPE composites. BioResources, v.5(2), p.661-671, 2010.

6. IBRAHIM, M. M.; DUFRESNE, A.; EL-ZAWAWY, W. K.; AGBLEVOR, F. A. Banana fibers and microfibrils as lignocellulosic reinforcements in polymer composites. Carbohydrate Polymers, v.81, p.811-819, 2010.

7. ALVES, C.; FERRÃO, P.M. C.; SILVA, A. J.;REIS,L.G.;FREITAS,M.;RODRIGUES, L. B.; ALVES, D. E. Ecodesign of automotive components making use of natural jute fiber composites. Journal of Cleaner Production, v.18, p.313-327, 2010.

8. DE ROSA, I. M.; SANTULLI, C.; SARASINI, F. Mechanical and thermal characterization of epoxy composites reinforced with random and quasiunidirectional untreated Phormium tenax leaf fibers. Materials \& Design, v.31, p.2397-2405, 2010.

9. MULINARI, D. R. Comportamento térmico, mecânico e morfológico dos compósitos de polietileno de alta densidade reforçados com fibras de celulose do bagaçodecana-de-açúcar. Tese(Doutorado em Engenharia Mecânica) - Universidade Estadual Paulista, Guaratinguetá, 2009.

10. BAKARE, I. O.; OKIEIMEN, F.E.; PAVITHRAN, C.; ABDUL KHALIL, H.P.S.; BRAHMAKUMAR, M. Mechanical and thermal properties of sisal fiber-reinforced rubber seedoilbased polyurethane composites. Materials \& Design, v.31, p.4274-4280, 2010.

11. ChERIANA, B. M.; LEÃO, A. L.; SOUZA, S. F.; THOMAS, S.; POTHAN, L. A.; KOTTAISAMY, M. Isolation of nanocellulose from pineapple leaf fibres by steam explosion. Carbohydrate Polymers, v.81, p.720-725, 2010.
12. LUO, S.; NETRAVALI, A. N. Interfacial and mechanical properties of environment-friendly "green" composites made from pineapple fibers and poly(hydroxybutyrate-co-valerate) resin. Journal of Materials Science, p.3709-3719, 2004.

13. ARIB, R. M. N.; SAPUAN, S. M.; AHMAD, M. M. H. M.; PARIDAH M. T.; KHAIRUL ZAMAN, H. M. D. Mechanical properties of pineapple leaf fibre reinforced polypropylene composites. Materials \& Design, v.27, p.391-396, 2006.

14. CANeVAROLO Jr., S. V. Ciência dos Polímeros. São Paulo: Artliber Editora. 2006.
Endereço para Correspondência: Daniella Regina Mulinari daniella.mulinari@foa.org.br Centro Universitário de Volta Redonda Campus Três Poços

Av. Paulo Erlei Alves Abrantes, $n^{\circ}$ 1325, Três Poços - Volta Redonda / RJ CEP: $27240-560$ 